\title{
Cosmology in the dark
}

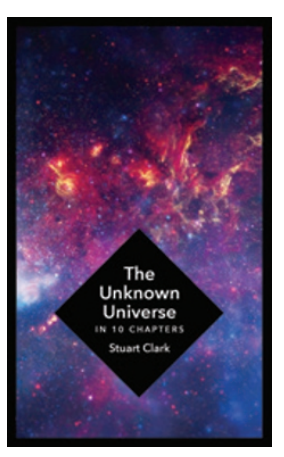

\author{
The Unknown \\ Universe: \\ A New Exploration \\ of Time, Space, and \\ Modern Cosmology \\ by Stuart Clark \\ HEAD OF ZEUS: 2015 \\ 288PP. $€ 16.99$
}

S tuart Clark's new book sweeps over you like a maelstrom of names, places and ideas. In The Unknown Universe, Clark sets out to describe the state of our knowledge - and lack thereof - about central questions of physics, astronomy and cosmology in fewer than 300 pages. Is our best model of the Universe simply a fudge held together by dark matter and threatened to be ripped apart by dark energy? Are black holes the places where Einstein's picture-perfect theory of general relativity metamorphoses into elusive quantum gravity? Is there such a thing as a single elapsed time since the Big Bang? And does string theory fulfil the basic definition of a falsifiable scientific hypothesis or is it simply a waste of time and effort on the part of a whole generation of bright scientists?

Clark attempts to frame these questions in their current setting, and begins with a journalistic account of a 2013 press conference held by the European Space Agency, during which images of the cosmic microwave background taken by the Planck spacecraft were presented. He then takes us on a journey to the dawn of the scientific revolution and starts unfolding the thread that links our understanding of the cosmos with that of intellectual giants such as Kepler and Newton. The book quickly settles into familiar historyof-science territory, recounting scientists' early attempts to explain the motion of comets and planets, and the birth of the Solar System.

The account is far from linear - we fast-forward to the mid-1980s discovery of a circumstellar disk that caused our Solar System to lose its privileged position in the grand scheme of things and vindicated Laplace's nebular hypothesis. One feels that the flow is interrupted somewhat by a digression to the Moon and theories of its formation, interspersed with factoids about natural nuclear reactors, lunar or terrestrial, along with basic facts on the architecture of the Solar System. But the account finds its thread again in the evolution of our ideas about the Sun and the stars as nuclear fusion machines that spew out chemical elements, our concept of the nature of light, and stellar spectroscopy. All bedrocks of modern astrophysics, and essential tools for measuring the properties of stars without in the author's own words - having to stick a thermometer into the Sun's core.

Clark offers a captivating description of the experiments that estimated the speed of light and the casting of entrenched scientific obsessions, such as the ether, into the dustbin of history. He then launches into twentieth-century science: how spiral nebulae turned out to be distant galaxies (island

\section{How Do You Find an Exoplanet?}

By John Asher Johnson

PRINCETON UNIVERSITY PRESS: 2016. 208PP. £24.95

This guide for planet hunters provides an accessible overview of the ongoing search for new alien worlds and our current understanding of how planets are formed.

The Unforgotten Sisters: Female Astronomers and Scientists before Caroline Herschel

By Gabriella Bernardi

SPRINGER PRAXIS BOOKS: 2016. 105PP. E62.99

Bernardi tells the stories of twenty-five female scientists, mostly astronomers and mathematicians, from Hypatia of Alexandria to Maria Gaetana Agnesi, the fictional heroes of Caroline Herschel. universes), rather than clouds within the confines of the Milky Way; general relativity; neutrons, neutrinos and the flat rotation curves of galaxies that ushered in one of the big mysteries of modern cosmology — dark matter. The book reaches a high point in these chapters, detailing how the scientific method was applied by various key figures who shaped modern science through debate, extrapolation, systematic experimental work and the occasional daydream. The story is accessible to the scientifically minded layperson.

The final chapters offer a light touch on the prospects for finding dark-matter particles, the merits of modified Newtonian dynamics, and attempts to reconcile theories of a seemingly flat, isotropic and thermally homogeneous Universe with one that is dominated by dark energy, and where galaxies cluster in vast filaments suspended at the edges of huge dark voids. Given the book's introduction, it is here that I had hoped to find a detailed analysis of cosmology's current travails. But these chapters are dotted with the author's accounts of his exchanges with other scientists that read more like a blog than a popular science book. Often I felt as though I'd chanced upon a wormhole that transported me back, against my will, from 2006 and the heady days of dark matter, to the 1600s to read about Kepler and his problems with Mars. Similarly, in the final chapter I was left wanting more of Hawking and his evaporating black holes, and perhaps an explanation of what really happened to the main protagonist from Interstellar.

Elsewhere in the book, names come and go as if they were quantum vacuum fluctuations: among others, Roger Penrose and Vahe Gurzadyan and their conformal cyclic cosmology. Also, non-cosmologists will grasp the concept of inflation, but its relation to primordial gravitational waves is perfunctorily described, and does not tie in well with the author's account of the purported detection of the waves' signatures by the BICEP 2 radio telescope. It is in such instances that Clark spreads himself too thinly. Although in places this is a readable account of the complexities of our Universe, one can't help but wonder if the author is as exasperated as we are about the failure of modern physics and cosmology to show us a clear way forward.

\section{REVIEWED BY YIANNIS TSAMIS}

Yiannis Tsamis is an astronomer who has worked at University College London and the European Southern Observatory. 